\title{
Weather and atmosphere observation with the ATOM all-sky camera
}

\author{
Felix Jankowsky a and Stefan Wagner \\ Landessternwarte Heidelberg, Königstuhl 12, 69117 Heidelberg, Germany
}

\begin{abstract}
The Automatic Telescope for Optical Monitoring (ATOM) for H.E.S.S. is an $75 \mathrm{~cm}$ optical telescope which operates fully automated. As there is no observer present during observation, an auxiliary all-sky camera serves as weather monitoring system. This device takes an all-sky image of the whole sky every three minutes. The gathered data then undergoes live-analysis by performing astrometric comparison with a theoretical night sky model, interpreting the absence of stars as cloud coverage. The sky monitor also serves as tool for a meteorological analysis of the observation site of the the upcoming Cherenkov Telescope Array. This overview covers design and benefits of the all-sky camera and additionally gives an introduction into current efforts to integrate the device into the atmosphere analysis programme of H.E.S.S.
\end{abstract}

\section{Introduction}

The Automatic Telescope for Optical Monitoring (ATOM) is a $75 \mathrm{~cm}$ optical telescope which forms part of the High Energy Stereoscopic System (H.E.S.S.) and is situated at the H.E.S.S. site in Göllschau, Namibia. It regularly monitors Very High Energy sources visible in optical wavelengths - mostly Active Galactic Nuclei - and serves both as multi-wavelength support instrument and alerts the H.E.S.S. Cherenkov telescopes in case a source shows higher-than-average flux. In general, observation runs resulting in significant detections are shorter compared to Cherenkov telescopes. Therefore, it can cover many more sources per night - typically 20 to 30 .

ATOM works fully automatic, meaning it does not need any manual intervention to perform its tasks. It schedules observation targets automatically, performs nightly operations with a robotic system and analyses the data using an automatic pipeline. This also means that no human observer is present during the night.

While Namibian weather is usually extraordinarily dry, rain can occur at the H.E.S.S. site during "rain season" in southern summer. As weather protection, a simple rain monitor would not be sufficient as it would trigger a telescope shut-down too late. Hence, the sky monitor was installed in 2008. It is a custom-built all-sky camera which observes the cloudiness of the sky and stops observation if the cloud coverage indicates that rain might start soon.

Section 2 gives an overview over the unique design of the sky monitor. Section 3 shows the benefits of equipping ATOM with this device and Sect. 4 gives an introduction into further use of the sky monitor for atmospheric characterisation.

\section{Device design}

The sky monitor - shown in Fig. 1 - was designed and built at Hamburger Sternwarte ${ }^{1}$. In its current form from 2008 it contains a $640 \times 480$ pixel camera placed behind

a e-mail: f . jankowsky@lsw. uni-heidelberg. de

1 www.hs.uni-hamburg.de a fish-eye lens and observing white light without any filter. It is unique among all-sky cameras as it possesses a protective lid which covers the lens whenever the device is not observing, preventing dust build-up. The lid also ensures protection from water, as it is kept closed whenever the rain sensor detects rain. It can therefore sustain consistent device performance over several months without the need for any maintenance, whereas all-sky monitors with unprotected glass domes need frequent cleaning to offer quantitatively reliable data - especially after every rainfall.

Two units are currently in use: one for regular operation of ATOM and another gathering comparison data for the Cherenkov Telescope Array site characterisation at its candidate-site in Aar, Southern Namibia.

The sky monitor is configured to take a 60 second exposure all-sky image every three minutes at time of writing. The data analysis follows the long-established method using astrometric comparison. In general, it extracts the position of all visible stars up to 5th magnitude and compares them with a theoretical model of the expected night-sky. If any expected stars are missing, it is assumed to be covered by clouds. Quality values ranging from 0 (worst) to 10 (best) are then assigned for different sectors of the sky based on the observed star visibility. An example for an analysed image is shown in Fig. 2.

\section{Benefits for telescope operation}

The original purpose of the sky monitor was to serve as a weather warning system. Whenever the sky quality distribution over the different sky sectors falls below a certain threshold, the sky monitor stops the telescope and closes the building. If at any point during night the weather conditions rise above said threshold, the telescope is activated and resumes observation. This threshold is defined by setting different requirements on different sectors, i.e. the zenith sector is more critical than the sectors at horizon. It can be adjusted easily for different sites characteristics.

The benefits of this weather monitoring can be compared with a mode in which the decision to observe

This is an Open Access article distributed under the terms of the Creative Commons Attribution License 4.0, which permits unrestricted use, distribution, and reproduction in any medium, provided the original work is properly cited. 


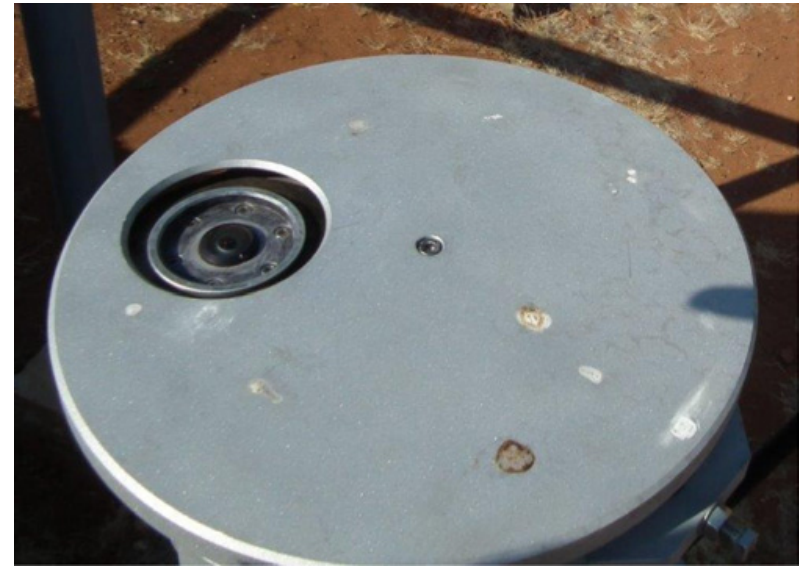

Figure 1. The sky monitor seen from above, with its protective lid turned for observation, showing the fish-eye lens.

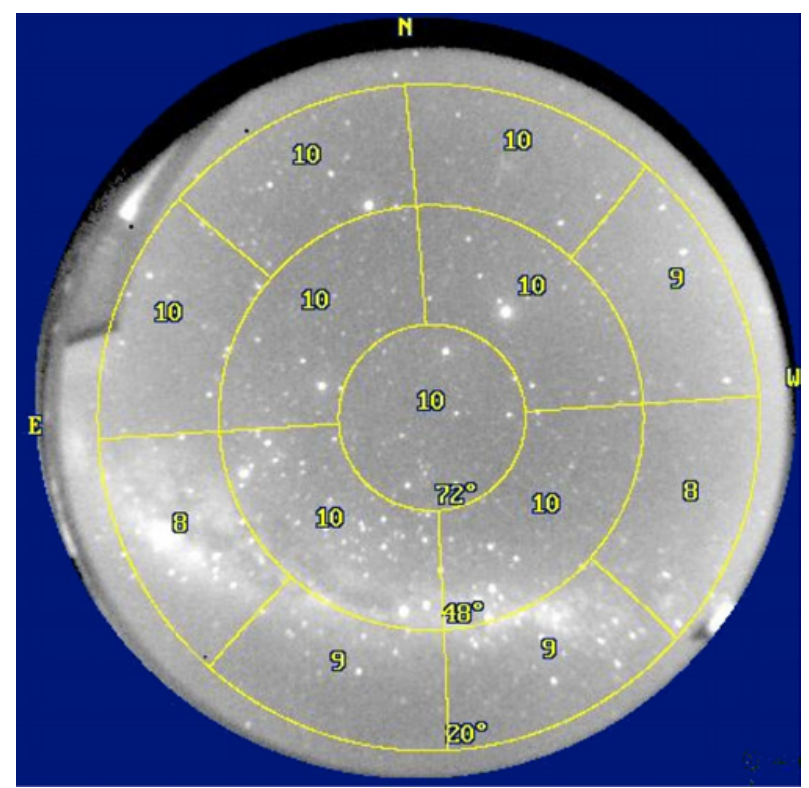

Figure 2. Example for an analysed sky monitor image, showing good quality values for all defined sectors.

or not is based on the weather during the evening. For such nights with a clear evening, cloud coverage at the H.E.S.S. site dropped below the bad weather threshold 303 times since the sky monitor has been installed. This does not necessitate the occurrence of rain nor does it mean that cloudiness stayed high for the whole night, but it does mean that without the sky monitor, there would have been a non-negligible chance for rain damaging the telescope.

On the other hand, the telescope gained 154 hours observation time during nights with cloudy evening. In this case the sky monitor noted the clouds clearing up and started observations accordingly.

As another benefit, the sky monitor also provides information on the distribution of clouds. This can be used for "smart-scheduling", i.e. the selection of an observation target which is currently in a visible part of the sky. For the H.E.S.S. site, the number of images taken with a certain number of covered sectors is shown in Fig. 3. As can be seen, there is a significant amount of time with partly

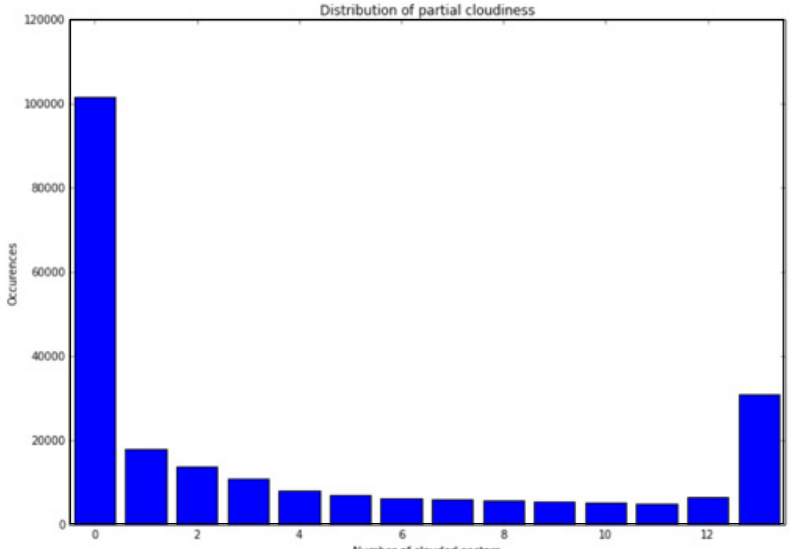

Figure 3. Number sky monitor images taken since its installation with a certain number of clouded sectors, the "clouded" threshold in this case being defined as $20 \%$ intransparency. It should be noted that the number of frames under full coverage is sligthly underestimated, as the sky monitor stops observation during rain.

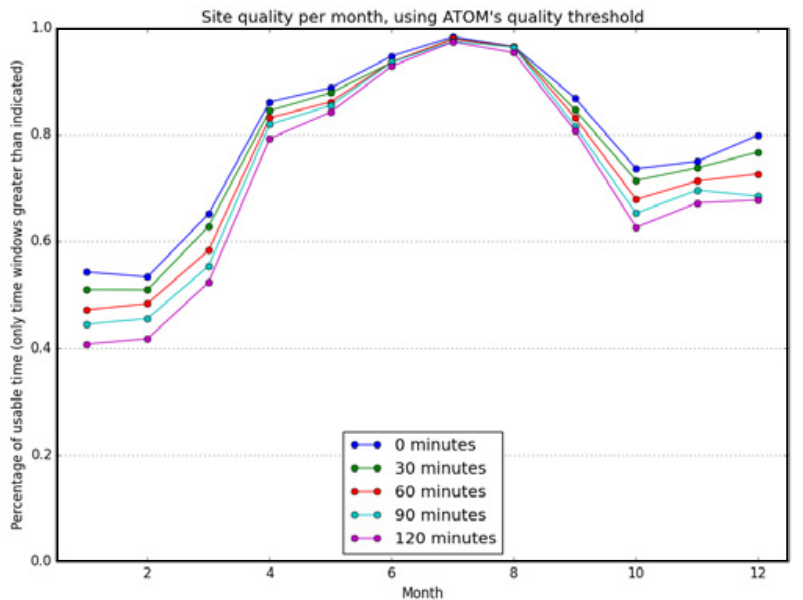

Figure 4. Percentage of observable time for different months at the H.E.S.S. site. As indicator for "observable" the cloudiness threshold employed for ATOM operations - which weighs clouds at zenith higher than those at horizon - was used. The different curves reflect the minimum time required to consider a period of good weather as observable, i.e. when the cloud clear up for less than the indicated amount of time, that duration is still counted as "bad weather".

clouded sky, which is potentially lost or gained observation time - although a more in-depth analysis than given here is required to quantify this effect.

\section{Weather and atmosphere analysis}

Clouds are usually the main reason for loss of observation time at any telescope site. Therefore, direct measurement of their occurrence is a prime target for any site characterisation campaign. Due to its short exposure time, wide field-of-view and its long-term stability, the sky monitor is an excellent tool for site characterisation. It is used as such for the site selection of the upcoming Cherenkov Telescope Array.

Figure 4 gives an exemplary analysis result using data from the ATOM sky monitor. The different-coloured 
curves show that only using an averaged value for large time-periods - e.g. a nightly bin - ignores that rapidly oscillating cloud-coverage would still cause any telescope to remain inactive, if the night's weather does not contain large enough time-windows of clear sky that accommodate at least one observation plus the time necessary for telescope preparation. Just taking a mean over the whole night would create the illusion that part of the night could be used for observations although that is clearly not the case.

For the H.E.S.S. site, it can be seen that while this effect becomes noticeable in the range above 60 minutes, the Namibian weather still tends to be quite stable over time.

To register a cloud pattern as "good weather" in Fig. 4, the requirements for operation of ATOM are applied. In this scheme, clouds at horizon are tolerated to a certain extend. Cloud coverage at zenith, however, needs to be minimal. This is a much more realistic requirement compared to a scenario in which the sky quality has to be above a certain value which is averaged over all sky sectors. Neither does the latter reflect that partial cloudiness might still be usable for observations in clear sections of the sky, nor does it take into account that especially for Cherenkov telescopes - the sectors closer to zenith are of greater importance than those at horizon, in addition to clouds not situated above the observatory posing no danger of rain.

Defining cloud pattern requirements for sky monitor data which well represent the properties of Cherenkov telescopes is not straightforward, as can also be seen in the following section.

\section{Detecting aerosols}

As is detailed in *corresponding HESS atmohead paper*, the biomass burning effect is a periodical appearance of an aerosol layer which severely inhibits the detection of Cherenkov light. As support for H.E.S.S.' Cherenkov Transparency measurement campaign, the possibility to use the sky monitor as an aerosol warning system has been examined.

This effort has faced severe challenges both for simple detection of aerosols as well as for differentiation from other types of atmospheric distortion. Many of these difficulties can be traced to the design of the sky monitor as simple cloud detection system, especially its low resolution and lack of colour information. In addition, the process of understanding the Cherenkov Transparency Coefficient, which has been used as the primary source to identify aerosols, is still ongoing.

The most promising solution at the time of writing is a large-scale photometry using the difference between the sky monitor sectors at the horizon and those at zenith. While the latter are generally unaffected by the biomass burning effect, the former do show a tendency to drop in sky monitor transparency. An example of this phenomenon is shown in Fig. 5.

However, a quick comparison of this sector difference with the Cherenkov Transparency Coefficient does not

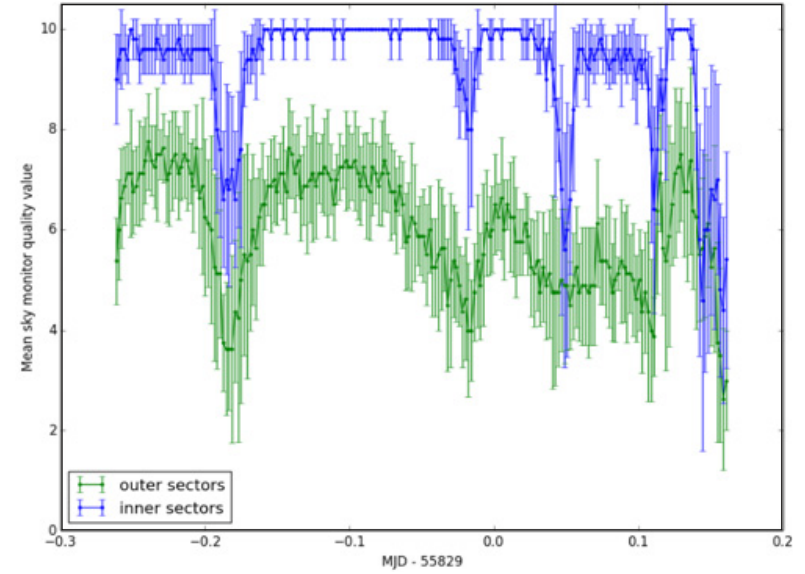

Figure 5. Exemplary night with biomass burning effect visible in form of lower sky monitor quality value in the sectors close to horizon (outer sectors), but high values in the ones at zenith (inner sectors). The bars indicate the spread of the quality value. Manual checks showed that the momentary drops in the inner sectors are due to passing clouds in an otherwise clear night.

show significant correlation although a trend is visible in September data, which usually is most affected by biomass burning. As can be seen, linking all-sky camera data with Cherenkov data is not straightforward. Still, investigation of several analysis and linking methods is ongoing.

\section{Conclusion}

The sky monitor has proven to be an excellent tool for its original purpose, the detection of clouds in the night sky. In this capacity, it is an invaluable tool for the automatic operation of ATOM, ensuring safe operations as well as that no usable observation time is lost. A by-product has been the collection of six years of weather data with a frequency of three minutes. Its unique design - which differentiates it from other all-sky cameras by protecting it against dust build-up and rain - enables use of the data archive for quantitative purposes.

However, first comparisons of sky monitor and H.E.S.S. data have shown that a direct correlation between the atmospheric transparency for both instruments should not be assumed. It has been proven especially challenging to reconstruct drops in Cherenkov transparency caused by aerosols. Investigation of this phenomenon is ongoing.

In addition, preparation for construction of an enhanced version of the sky monitor is underway. It will retain the mechanical advantages of the current model, but feature enhanced optical properties, i.e. higher resolution and colour imaging. First tests showed that the new sky monitor can detect stars of at least 8th magnitude in 15 second exposures. It will not only act as support instrument for ATOM by measuring extinction properties of different regions of the sky, but is also designed as a tool for precise atmospheric characterisation. These results will offer further possibilities for analysis of H.E.S.S. data. 